\title{
МЕСТО ОПЕРАЦИИ ПЛАСТИКИ АОРТАЛЬНОГО КЛАПАНА АУТОПЕРИКАРДОМ ПО ОЗАКИ В ЛЕЧЕНИИ ПРИОБРЕТЕННЫХ АОРТАЛЬНЫХ ПОРОКОВ
}

\section{THE PLACE OF THE OZAKI OPERATION IN THE SURGICAL THERAPY OF AORTIC VALVE FAILURE}

A. Moiseev

Summary. The article is devoted to the review, long-time results and modifications of aortic valve reconstruction with glutaraldehyde-treated autologous pericardium.

Keywords: aortic valve; aortic valve reconstruction.
Моисеев Алексей Александрович

alexi.moiseev@gmail.com

Аспирант, ФГАОУ ВО «Российский начиональный исследовательский медицинский университет имени Н.И. Пирогова» Министерства здравоохранения Российской Федерации

Аннотация. Статья представляет обзор современной литературы по оперативной технике, отдаленным результатам и модификациям пластики аортального клапана аутоперикардом по методике Озаки.

Ключевые слова: аортальный клапан; реконструкция аортального клапана; операция Озаки.

\section{Введение}

$\Pi$ атология аортального клапана является самой распространенной среди клапанных пороков, их доля составляет 44,3\%. Лидирующее место принадлежит аортальному стенозу, встречающемуся с частотой 33,9\% согласно исследованию Euro Heart Survey (1). В течение длительного времени протезирование аортального клапана является золотым стандартом лечения патологии приобретенных изолированных аортальных пороков. За год в мире выполняется 280000 вмешательств (2). В группе пациентов с аортальным стенозом высокого операционного риска показано преимущество выполнения транскатетерного протезирования аортального клапана (3).

На сегодняшний день существует широчайший выбор технологий замены аортального клапана и корня аорты. Несмотря на это идеальный протез аортального клапана остается лишь теоретической моделью, имеющей те же гемодинамические и биологические характеристики, что и человеческий клапан, не склонной к тромбогенезу и развитию инфекционных осложнений. Воплотить данную модель в реальность к настоящему времени не удалось (4).

Протезирование аортального клапана сопряжено с рядом специфических осложнений. В исследовании (5) была выявлена повышенная госпитальная летальность при имплантации протезов клапана малого диа- метра (19 мм) в группе пациентов с узким корнем аорты. В работе (6), посвященной исследованию отдаленных результатов хирургического лечения инфекционного эндокардита аортального клапана, осложненного формированием абсцесса корня аорты, отмечена высокая частота рецидивирования инфекции, в 10\% случаев потребовавшей проведения повторного оперативного вмешательства.

\section{Современное состояние реконструкции аортального к^апана}

Попытки реконструкции аортального клапана предпринимаются в течение длительного времени. Первая публикация принадлежит Taylor, 1958 г. и сводится к наружной субкоронарной шовной пластике расширенного кольца аортального клапана на работаюшем сердце (7). Первая в условиях искусственного кровообращения техника пластики аортального клапана опубликована Cabrol в 1966 г., ее суть в выполнении субкомиссуральной пликации створок аортального клапана, за счет которой достигается редукция диаметра дилатированного кольца (8). Обе методики имеют существенный недостаток, связанный с ранним, в сроки до 12 месяцев, рецидивированием и прогрессированием аортальной недостаточности. Кроме того, оба варианта пластики являются высокооператорозависимыми, поскольку необходимая степень сужения аортального кольца субьективна, а в случае Taylor вовсе практически недоступна для объективной интраоперационной оценки. 
В 2006 г. в работах Lansac и соавт. описана наружная пластика аортального клапана гибким кольцом в субкоронарной позиции, позволяющая с удовлетворительными долгосрочными результатами, как изолированно, так и в сочетании с супракоронарным протезированием восходящей аорты при ее аневризме, либо в сочетании с операцией Yakoub при аневризме корня аорты, устранять аортальную недостаточность, обеспечивая 7-летнюю свободу от повторных вмешательств на аортальном клапане в $92 \%$ случаев $(9,10)$.

В современных рекомендациях по лечению клапанной болезни сердца реконструкция аортального клапана по David либо Yakoub в сочетании с аннулопластикой считается показанной в группе молодых пациентов при достаточном уровне подготовки хирургической бригады (11).

Несмотря на это большая часть вмешательств на аортальном клапане сводится к его протезированию. Так, например, в Германии в 2018 г. выполнено 10022 операций коррекции его пороков. Из них реконструкций - 193 (12). По всей видимости, это связано с невозможностью пластики значительно измененных створок, в частности, при тяжелом аортальном стенозе.

\section{Реконструкция \\ аорта^ьного к^апана по Озаки}

Пластика створок аортального клапана по Озаки представляет собой новый метод реконструкции створок аортального клапана обработанным аутоперикардом, описанный в 2010 г. проф. S. Ozaki (13). Это инновационный метод пластики аортального клапана, являющийся альтернативой выполнению протезирования. Преимуществом метода является отсутствие имплантируемых в корень аорты искусственных материалов.

Впервые техника пластика створок аортального клапана аутоперикардом была впервые описана Duran в 1995 г (14). Отличие процедуры Озаки заключается в стандартизации выполнения последней.

Суть метода заключается в замещении иссеченных створок аортального клапана обработанным глутаральдегидом аутоперикардом. Нео-створки выкраиваются с использованием стандартного трафарета. Размер створок определяется на основе измерения межкомиссурального расстояния стандартным измерителем.

Створки фиксируются в корне аорты непрерывным швом в обеих направлениях, начиная с нижней точки соответствующего коронарного синуса. Оперативным доступом является полная срединная стернотомия, что обусловлено необходимостью забора аутоперикарда (13).
В 2018 г. Ozaki и соавт. опубликован анализ отдаленных результатов выполнения пластики у 850 пациентов со средним возрастом в 71 г (62-78 л) (15). Основным показанием к операции был аортальный стеноз (62,8\%). Остальная группа включала больных с аортальной недостаточностью (29,9\%), сочетанным пороком $(7,2 \%)$, инфекционным эндокардитом (2,2\%).

В 57\% случаев выполнена изолированная процедура Озаки, в остальных - сочетанные вмешательства - симультанное протезирование восходящей аорты (13,3\%), АКШ (9,9\%). В случае изолированных вмешательств время искусственного кровообращения 106,8 $\pm 27,5$ мин, время ишемии 150,4 $\pm 30,4$ мин.

В исследовании проанализированы 7-летние результаты выполнения процедуры Озаки. Отмечена выживаемость в $85,9 \%$. Смертность в $80 \%$ случаев имеет экстракардиальные причины.

Отмечена стабильность градиента в послеоперационном периоде на уровне 19,5 $\pm 10,3$ мм. рт.ст., аортальная недостаточность 2 ст. и выше - 17 пациентов (0,02\%).

Основным осложнением среди прооперированных пациентов, требующих повторного вмешательства, был инфекционный эндокардит (0,3\% случаев - 15 пациентов).

Группой Ozaki и соавт. выполнен в 2014 г. анализ эффективности метода в группе пациентов старше 80 лет (16). В ходе 6-летнего наблюдения отмечен стабильный градиент на уровне 15 мм рт. Ст. несмотря на узкий корень аорты в 90,7\% случаев. Выживаемость составила 87\% с исключительно некардиальной летальностью.

\section{МоАификашии метолики Озаки}

Развитием метода стало выполнение Nguyen и соавт. операции из министернотомии с торакоскопическим забором аутоперикарда и периферическим подключением аппарата искусственного кровообращения (17). Rosseykin и соавт. доказали, что основным статистически значимым преимуществом в этом случае является снижение суммарной кровопотери (18).

Еще одна модификация операции Озаки была разработана Komarov с соавт. и представляет собой гибрид операции Дэвида и Бенталла для одновременной коррекции аортального порока и протезирования аорты. Модификация заключается в имплантации створок из аутоперикарда в сосудистый протез с последующим замещением корня аорты и ее восходящего отдела таким графтом (19). 


\section{Зак^ючение}

Методика проведения пластики аортального клапана аутоперикардом по Озаки имеет надежную физиологическую базу, заключающуюся в увеличении высоты зоны коаптации и сохранении естественного движения кольца аортального клапана. Кроме того, обработанные глутаральдегидом аутоперикардиальные нео-створки имеют повышенную устойчивость к растяжению. В связи с этим указанный метод является достойной альтернативой протезированию аортального клапана, позволяю- щей корригировать практически весь спектр патологии аортального клапана.

\section{КонФАикт интересов}

Автор заявляет об отсутствии конфликта интересов.

\section{Информашия о финансировании}

Финансирование исследования за счет средств автоpa.

\section{ЛИТЕРАТУРА}

1. lung B., Baron G., Butchart E. G., Delahaye F., Gohlke-Bдrwolf C., Levang 0. W., et al. A prospective survey of patients with valvular heart disease in Europe: The Euro Heart Survey on valvular heart disease. Eur Heart J. 2003;24(13):1231-43.

2. Pibarot P., Dumesnil J.G. Prosthetic heart valves: Selection of the optimal prosthesis and long-term management. Circulation. 2009;119(7):1034-48.

3. Smith C. R., Leon M. B., Mack M. J., Miller D. C., Moses J. W., Svensson L. G., et al. Transcatheter versus Surgical Aortic-Valve Replacement in High-Risk Patients. N Engl J Med. 2011;364(23):2187-98.

4. Hoffmann G., Lutter G., Cremer J. Durability of bioprosthetic cardiac valves. Dtsch Arztebl. 2008;105(8):143-8.

5. Adams D. H., Chen R. H., Kadner A, Aranki S. F., Allred E. N., Cohn L. H., et al. Impact of small prosthetic valve size on operative mortality in elderly patients after aortic valve replacement for aortic stenosis: Does gender matter? J Thorac Cardiovasc Surg. 1999;118(5):815-22.

6. Croon S. I., Angkasuwan A., van Straten A. H., Khamooshian A., Elenbaas T.W., Soliman-Hamad MA. Surgical treatment and long-term outcome of aortic valve endocarditis with periannular abscess. Netherlands Hear J. 2020;28(6):345-53.

7. Taylor W. J., Thrower W. B., Black H., Harken D. E. The surgical correction of aortic insufficiency by circumclusion. J Thorac Surg. United States; 1958 Feb;35(2):192205 passim.

8. Cabrol C, Cabrol A, Guiraudon G, Bertrand M. [Treatment of aortic insufficiency by means of aortic annuloplasty]. Arch Mal CoeurVaiss. France; 1966 Sep;59(9):130512.

9. Lansac E., Di Centa I., Sleilaty G., Lejeune S., Berrebi A., Zacek P., et al. Remodeling root repair with an external aortic ring annuloplasty. J Thorac Cardiovasc Surg. Elsevier Inc.; 2017;153(5):1033-42.

10. Lansac E., Di Centa I., Raoux F., Bulman-Fleming N., Ranga A., Abed A., et al. An expansible aortic ring for a physiological approach to conservative aortic valve surgery. J Thorac Cardiovasc Surg. 2009;138(3):718-24.

11. Baumgartner H., Falk V., Bax J. J., De Bonis M., Hamm C., Holm P. J., et al. 2017 ESC/EACTS Guidelines for the management of valvular heart disease. Vol. 38, European Heart Journal. 2017. 2739-2786 p.

12. Beckmann A., Meyer R., Lewandowski J., Markewitz A., Harringer W. German heart surgery report 2018: The annual updated registry of the german society for thoracic and cardiovascular surgery. Thorac Cardiovasc Surg. 2019;67(5):331-44.

13. Ozaki S, Kawase I, Yamashita H, Uchida S, Nozawa Y, Matsuyama T, et al. Aortic valve reconstruction using self-developed aortic valve plasty system in aortic valve disease. Interact Cardiovasc Thorac Surg. 2011;12(4):550-3.

14. Duran C. M.G., Gometza B., Kumar N, Gallo R, Martin-Duran R. Aortic valve replacement with freehand autologous pericardium. J Thorac Cardiovasc Surg. 1995;110(2):511-6.

15. Ozaki S, Kawase I, Yamashita H, Uchida S, Takatoh M, Kiyohara N. Midterm outcomes after aortic valve neocuspidization with glutaraldehyde-treated autologous pericardium. JThorac Cardiovasc Surg. Elsevier Inc.; 2018;155(6):2379-87.

16. Ozaki S, Kawase I, Yamashita H, Uchida S, Nozawa Y, Takatoh M, et al. Aortic valve reconstruction using autologous pericardium for ages over 80 years. Asian Cardiovasc Thorac Ann. 2014;22(8):903-8.

17. Nguyen D. H., Vo A. T., Le K. M., Vu T. T., Nguyen T. T., Vu T. T., et al. Minimally Invasive Ozaki Procedure in Aortic Valve Disease: The Preliminary Results. Innov Technol Tech Cardiothorac Vasc Surg. 2018;13(5):332-7.

18. Россейкин Е. В., Кобзев Е. Е., Базылев В. В. Операция Оzаkі из мини-доступа // Ангиология и сосудистая хирургия. 2019; № 3 с 142-155.

19. Komarov R, Chernov I, Enginoev S, Michel Pompeu B.0 S6, Tarasov D. The russian conduit — combining bentall and ozaki procedures for concomitant ascending aorta replacement and aortic valve neocuspidization. Brazilian J Cardiovasc Surg. 2019;34(5):618-23. 